\title{
Acciones pedagógicas y su incidencia en el manejo de los recursos sólidos a través de la IEP ${ }^{1}$ \\ Pedagogical actions and their incidence in the management of solid resources
}

DOI: http://dx.doi.org/10.17981/cultedusoc.9.3.2018.02

Artículo de investigación. Fecha de recepción: 15/06/2018. Fecha de aceptación: 27/11/2018

\author{
Jaime Arteta Saltarín²; \\ Yormaris De La Ossa-Cárdenas; Narlys González-Zarco; \\ Catalina Miranda-Gutiérrez y Marelvis Suarez ${ }^{3}$ \\ IED San José, sede Principal (Colombia) \\ judith-gon@hotmaill.com
}

Para citar este artículo:

Arteta, J., De La Ossa-Cárdenas, Y., González-Zarco, N., Miranda-Gutiérrez, C. y Suarez, M. (2018). Acciones pedagógicas y su incidencia en el manejo de los recursos sólidos a través de la IEP. Cultura. Educación y Sociedad 9(3), 19-24. DOI: http://dx.doi.org/10.17981/ cultedusoc.9.3.2018.02

\section{Resumen}

Una de las grandes dificultades que se presentan en las instituciones educativas, es el inadecuado manejo de los residuos sólidos en los estudiantes por razones variadas. Por tal motivo el presente estudio, buscó realizar una intervención apoyada en TIC, para implementar en los estudiantes una mentalidad amigable con el ambiente, inculcando a los estudiantes sentido de respeto y cuidado con el entorno de la institución y el medio ambiente, principalmente en la recolección y clasificación de los residuos generados en su institución. Metodológicamente se orientó bajo un enfoque cualitativo, desde un diseño descriptivo, donde se utilizaron los instrumentos de formato de observación participante y diario de campo. La muestra estuvo conformada por 250 estudiantes de la jornada de la mañana de la Institución Educativo Departamental San José Sede $N^{\circ} 4$. Los resultados demuestran que, una vez realizada la implementación de la estrategia, se logró impartir en los estudiantes un cambio, sobre todo evidenciado en los horarios de recreo donde se reflejó una mejoría en el manejo de los residuos sólidos.

Palabras clave: Acciones pedagógicas, residuos sólidos, medio ambiente, TIC.

\section{Abstract}

One of the great difficulties that occur in educational institutions, is the inadequate management of solid waste in students for various reasons. For this reason the present study, sought to perform an intervention supported by ICT, to implement in the students a friendly mentality with the environment, instilling to students a sense of respect and care with the environment of the institution and the environment, mainly in the collection and classification of the waste generated in your institution. Methodologically, it was oriented under a qualitative approach, from a descriptive design, where the instruments of participant observation format and field diary were used. The sample consisted of 250 students of the morning session of the Departmental Education Institution San José Sede No. 4. The results show that, once the implementation of the strategy, it was possible to impart a change to the students, especially evidenced in the schedules of recreation where an improvement in the management of solid waste was reflected.

Keywords: Pedagogical actions, solid waste, environmental, TIC.

\footnotetext{
1 Este artículo ha sido derivado del Programa de Fortalecimiento de la Cultura Ciudadana y Democrática CT+I a través de la IEP apoyada en TIC en el Departamento de Magdalena: CICLON
}

2 Líder del Grupo de Investigación "Medio Ambiente".

3 Docentes miembros del Grupo de Investigación "Medio Ambiente", de la IED San José, sede Principal.

- The author; licensee Universidad de la Costa - CUC 


\section{Introducción}

La situación medioambiental actual, es un tema que compete a todas las esferas de la sociedad y a las personas de todas las edades. Uno de los problemas más complejos es el manejo mundial de los residuos sólidos (Herrera-Mendoza, Ramírez, M., y Acuña, M., 2016), ya que el patrón consumista que prima en la sociedad, propicia el uso de productos que se utilizan en poco tiempo, pero que sus residuos permanecen en la tierra por muchos años, sobre todo cuando no se da un buen manejo de ellos, como es el caso de las bolsas plásticas que se obtienen cuando se va al supermercado, las bolsas y botellas cuando se toma agua, alimentos empacados, etc.; los desechos de plástico, vidrio y metal no desaparecen fácilmente (Hesperian, 2011)

En Colombia el 7.5\% de los rellenos sanitarios de país ya se les acabó su vida útil y a un $15 \%$ le quedan menos de 3 años de utilidad. (Revista Semana, 2018). Las basuras se están convirtiendo en un problema grave para el país; muchas familias viven cerca a rellenos sanitarios o acumulan basura por no existir un buen sistema de recolección de residuos, lo que permite la proliferación de plagas, insectos, animales y como consecuencia se ocasionan enfermedades como salpullidos en la piel, problemas estomacales, conjuntivitis, entre otras. Están también las personas que viven cerca de ríos, lagos y otras fuentes de agua, que están siendo altamente contaminadas con residuos sólidos y que utilizan esta agua para sus labores diarias, produciendo consecuencia a su salud (Crissien-Borrero, Cortés-Peña, \& Herrera-Mendoza, 2016).

Por otra parte, se encuentra encendida la alerta por la gran cantidad de residuos electrónicos, incluyendo computadores, impresoras y sobre todo celulares que han crecido de manera exponencial durante las dos últimas décadas, pues cuando terminan su vida útil muchos residuos electrónicos terminan en la basura común, no teniendo en cuenta las sustancias peligrosas que se encuentran en su composición como el arsénico y el plomo, que pueden entrar en contacto con el terreno y filtrarse incluso a las aguas subterráneas. (UNESCO, 2010).

En las instituciones educativas se halla una representación de la sociedad en menor medida, convergen costumbres, conocimientos, razones y comportamientos (Domínguez, 2009) de cada estudiante, docente y administrativo que conforma la comunidad educativa. Por esta razón también se evidencia el problema del manejo de los residuos sólidos, sobre todo al terminar el momento del descanso, donde en múltiples colegios, puede observarse el patio sucio y lleno de basura, así como también entre las clases, con papeles por todo el salón (Herrera-Mendoza, Acuña y Gil, 2017).

Expertos en el tema del medio ambiente "coinciden en reconocer la educación como la vía más expedita para generar conciencia y fomentar comportamientos responsables frente al manejo sostenible del ambiente" (Ministerio de Educación Nacional, 2005) y es a partir de este supuesto, se proponen en Colombia los PRAEs, o proyectos ambientales escolares, pensándolos como una estrategia que posibilita el estudio de la problemática ambiental local, y que contribuyera a la búsqueda de soluciones desde los miembros de la comunidad educativa (Sistema Nacional Ambiental-SINA, 2016), sobre todo desde los estudiantes, para inculcar su conexión, valoración y respeto hacia la naturaleza.

Es importante en el marco del presente artículo, precisar cómo se concibe el término residuos sólidos, a continuación, se muestra conceptualmente este aspecto.

\section{Residuos sólidos}

Los residuos sólidos son aquellas sustancias, productos y subproductos en estado sólido o semisólido, resultante del consumo 
o uso de un bien, a partir de actividades domésticas, industriales, institucionales y comerciales (Observatorio ambiental de Bogotá, 2015); estos deben manejarse en virtud de lo establecido en la normatividad de cada país, con la intención de minimizar los residuos, reciclarlos, reaprovecharlos, almacenarlos y disponerlos de acuerdo a su composición de forma correcta (Tejada, 2006).

Los residuos sólidos pueden ser aprovechables, con lo que normalmente se practica bien sea el reciclaje, la reutilización, o incineración con recuperación de energía, entre otros, volviendo al ciclo económico productivo y generando ingresos. También están los no aprovechables o denominamos basuras como tal, de los cuales se realiza un tratamiento, se debe disponer de ellos de forma controlada, para generar un mínimo impacto en el ambiente con su manejo (Penagos, Adarraga, Aguas y Molina, 2011).

En las escuelas, por su composición puede manejarse los residuos sólidos aprovechables, así también los estudiantes pueden reciclar y aprender a disponer de los desechos que producen diariamente.

\section{Metodología}

Para llevar a cabo el presente proyecto se definió el tipo de investigación cualitativa, desde un diseño descriptivo. La investigación cualitativa busca conocer e interpretar la realidad de los participantes a través de sus propias experiencias (Meza, Arrieta y Noli, 2018), entregando una información subjetiva del fenómeno de estudio (Hernández, Fernández, y Baptista, 2010).

\section{Población y muestra}

Los estudios con un diseño descriptivo están encaminados a especificar las características de la población sujeto de estudio o los fenómenos que sean sometidos a análisis (Hernández, Fernández, y Baptista,
2010). En cuanto a la población beneficiada con el estudio, se tomó una muestra representativa de doscientos cincuenta (250) estudiantes de Institución Educativo Departamental San José Sede $N^{\circ} 4$, distribuidos en los cursos de la jornada de la mañana, se realizó un muestreo de tipo intencional, escogiendo los estudiantes que se encontraban de preescolar a quinto grado, para observar la distribución de la muestra, ver la Tabla 1.

TABLA 1

Distribución de la población

\begin{tabular}{cc}
\hline Grado & Número de Estudiantes \\
\hline Preescolar & 86 \\
Primero & 30 \\
Segundo & 39 \\
Tercero & 28 \\
Cuarto & 33 \\
Quinto & 34 \\
TOTAL & $\mathbf{2 5 0}$ \\
\hline
\end{tabular}

Fuente: elaboración propia.

\section{Instrumentos}

Para la recogida de datos se utilizó un formato de observación participante y se tomaron anotaciones en un diario de campo, estos son instrumentos de recolección de datos, propios de la investigación cualitativa por la riqueza de información y vivencias que implican. En el diario de campo se registran y sistematizan los elementos obtenidos donde el contacto con las personas del contexto, modulan las reflexiones y conclusiones que se pueden extraer (Sáenz, 2017). El docente tomó un rol activo en el proceso de investigación, donde no solo estuvo como espectador del proceso de implementación, sino que también participó del mismo, registrando cada elemento observado con relación a la variable de estudio. 


\section{Procedimiento}

La metodología del proyecto de investigación se realizó a partir de fases, que comenzaron con el diagnóstico hasta la implementación y evaluación. Fue importante en el proceso incluir el uso de las TIC y articular la indagación al plan de estudios de las asignaturas, transversalizando las áreas de conocimiento. No obstante, se hace énfasis en las que estudian directamente el tema en mención, como las sociales y naturales, esto permite generar una trasformación social y educativa a través del aprendizaje en contexto, involucrando intereses, motivaciones y realidades de los estudiantes para generar conocimiento científico. Las fases fueron:

Fase 1: los docentes realizaron un diagnóstico, según los criterios de evaluación de las pruebas por competencias para identificar las dificultades específicas que presentaba la población en los procesos de manejo de residuos sólidos.

Fase 2: diseño de estrategias pedagógicas basadas en la investigación y las TIC transversales a las diferentes áreas de estudio, en especial a las que tratan el tema ambiental de acuerdo a las necesidades de la población. Fase 3: implementación y transversalización de las estrategias en el aula de clase por parte del cuerpo docente.

Fase 4: análisis e interpretación de los resultados.

Fase 5: Apropiación social sobre los conocimientos generados en materia de estrategias pedagógicas orientadas al buen manejo de los residuos sólidos.

\section{Resultados}

En la tabla 2, se observan los principales hallazgos del estudio por fases.

TABla 2

Resultados por fases

\begin{tabular}{ll}
\hline \multicolumn{1}{c}{ Fase } & \multicolumn{1}{c}{ Hallazgos importantes } \\
\hline & $\begin{array}{l}\text { Los estudiantes presentaban dificultades específicas sobre el buen y correcto manejo de los } \\
\text { residuos sólidos, se encontró que una gran parte de los estudiantes se encuentran informados } \\
\text { sobre la problemática ambiental por la que está pasando el planeta y son conscientes de que }\end{array}$ \\
Fase 1- & en la Institución se requiere una intervención para reducir los residuos sólidos dejados en \\
Diagnóstico & el suelo luego del tiempo de receso estudiantil, sin embargo, no aplican tales conocimientos \\
& para evitar perjudicar su entorno escolar; la mayoría de los observados no manejaban la \\
& información pertinente para clasificar de manera correcta los residuos sólidos en las canecas \\
de color respectivas.
\end{tabular}

Fuente: elaboración propia. 


\section{Discusión}

La implementación de un programa que propicie la educación ambiental resulta ser una muy buena alternativa para preservar el orden y la limpieza en el entorno escolar y además para concientizar a los estudiantes sobre la problemática a mbiental mundial y hacerlos entender que ellos son parte de ella y que por lo tanto deben colaborar a la reducción de la misma (Espejel, A. \& Flores A., 2012; Castillo, 2010; Herrera-Mendoza y Bravo, 2013), esto pudo corroborarse en la Institución Educativa Departamental San José Sede $\mathrm{N}^{\circ} 4$, específicamente con los 250 estudiantes participantes de las estrategias en el manejo de residuos sólidos.

Se evidenció que la institución presentaba dificultades marcadas en las competencias relacionadas con el buen manejo de los recursos sólidos, identificando que los esquemas de educación tradicional son poco efectivos incluso para los temas medioambientales porque no generan motivación en el estudiante, lo cual es un componente necesario para el desarrollo del aprendizaje.

Integrar a los estudiantes en las estrategias y jornadas donde en medio del juego desarrollaban conocimientos frente a su papel activo en el manejo de residuos y aún más cuando se apoyaban en las TIC para indagar sobre la problemática actual y soluciones, generó un impacto positivo en la comunidad educativa, involucrando a los grados escolares más pequeños, mediante la acción y la formación.

\section{Referencias}

Cabello-Eras, J. (2016). Acercamiento a la producción más limpia como estrategia de gestión ambiental. International Journal of Management Sciences and Operations Research, 1(1), 4-7. Recuperado de http://ijmsoridi.com/index.php/ ijmsor/article/view/71
Cortés-Peña, O. (2016). Sustainable development in synergistic relationship with pro-ambient behavior and fair trade. International Journal of Management Sciences and Operations Research, 1(1), 54-58. Retrieved from http://ijmsoridi. com/index.php/ijmsor/article/view/78

Crissien-Borrero, T., Cortés-Peña, O. y Herrera-Mendoza, K. (2016). Pro-Environmental Assessment and Sustainable Consumption of Household Public Services in Barranquilla Colombia. The European Proceedings of Social \& Behavioural Sciences - EpSBS, 17, 434440. DOI: http://dx.doi.org/10.15405/ epsbs. 2016.11

Domínguez, S. (2009). Las instituciones educativas en la representación social de la ciencia en estudiantes de pregrado. Interamerican Journal of Psychology, 43(3), 456-465.

Herrera, K. (2011). La crisis medioambiental. Una mirada desde la psicología. Barranquilla: Educosta.

Herrera-Mendoza, K., Acuña, M. y Gil, L. (2017). Motivación de jóvenes hacia el uso de teléfonos celulares. Revista encuentros, 15(1). 91-105.

Herrera-Mendoza, K., Ramírez, M. y Acuña, M. (2016). Actitud y conducta proecológica en jóvenes universitarios. Revista Opción, 32(13). 456-477.

Herrera-Mendoza, K. y Bravo, E. (2013). Perspectiva de la ecología en la comprensión de los comportamientos ambientales. Revista Omnia, 19(3). 20-30.

Martínez, R. (2010). La importancia de la educación ambiental ante la problemática actual. Revista Electrónica Educare, $X I V(1), 97-111$.

Meza, A., Arrieta, M. y Noli S. (2018). Análisis de la conciliación extrajudicial civil en la Costa Atlántica colombiana. Jurídicas CUC, 14(1). 187-210. DOI: http://dx.doi.org/10.17981/juridcuc.14.1.2018.9 
Nieves, J. Y Salazar, F. (2016). e-Supply chain management para una empresa de tecnología. International Journal of Management Sciences and Operations Research (IJMSOR), 1(1), 31-34. Recuperado de http://ijmsoridi.com/index. php/ijmsor/article/view/74

Observatorio ambiental de Bogotá. (2015). Manejo Residuos Sólidos. Recuperado de http://oab2.ambientebogota.gov.co/ es/educacion-ambiental/ayuda-de-tareas/manejo-residuos-solidos

Organización Hesperian. (2011). Guía comunitaria para la salud ambiental. Desechos sólidos: cómo convertir un riesgo para la salud en un recurso. Recuperado de http://hesperian.org/wp-content/ uploads/pdf/es_cgeh_2011/es_ cgeh_2011_cap18.pdf

Penagos J., Adarraga J., Aguas D. y Molina, E. (2011). Reducción de los residuos sólidos orgánicos en Colombia por medio del compostaje líquido. Revista Ingeniare. (11). 37-44. https://doi. org/10.18041/1909-2458/ingeniare. 11.643

República de Colombia. Ministerio de Educación Nacional. (2005). Educación Ambiental: construir educación y país. Bogotá, D.C.: Altablero. Recuperado de https://www.mineducacion.gov.co/1621/ article-90891.html
Revista Semana. (11 de mayo, 2018). Basura a punto de explotar. [Sección Impacto]. Recuperado de https://sostenibilidad.semana.com/impacto/articulo/manejo-deresiduos-en-colombia-es-una-bomba-apunto-de-estallar/40963

Sáenz, J. (2017). Investigación educativa. Fundamentos teóricos, procesos y elementos práctico con ejemplos, esencial para TFC, TFM y tesis. Madrid: UNED publicaciones.

SINA. (2016). Los proyectos ambientales escolares-PRAE, en Colombia: viveros de la nueva ciudadanía ambiental de un pais que se construye en el escenario del posconflicto y la paz. Recuperado de http:// www.minambiente.gov.co/images/OrdenamientoAmbientalTerritorialyCoordi-

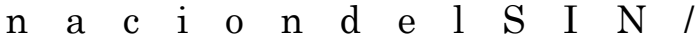
pdf/VII_Encuentro_Nacional_de_ Educaci\%C3\%B3n_Ambiental/PRAE.pdf

Tejada, G. (2006). Manejo de residuos sólidos en la ciudad de Tacna. Revista ciencia y desarrollo. (10). Recuperado de http://revistas.unjbg.edu.pe/index.php/ CYD/article/view/205/182

UNESCO. (2010). Los residuos electrónicos: Un desafío para la Sociedad del Conocimiento en América Latina y el Caribe. Recuperado de http://www.unesco.org. uy/ci/fileadmin/comunicacion-informacion/LibroE-Basura-web.pdf 